\title{
Sensitivity of Aquarius Active and Passive Measurements Temporal Covariability to Land Surface Characteristics
}

\author{
María Piles, Member, IEEE, Kaighin A. McColl, Student Member, IEEE, Dara Entekhabi, Fellow, IEEE, \\ Narendra Das, and Miriam Pablos, Student Member, IEEE
}

\begin{abstract}
Active and passive microwave observations over land are affected by surface characteristics in different ways. L-band radar backscatter and radiometer measurements each have distinct advantages and problematic issues when applied to surface soil moisture estimation. Spaceborne radiometry has the advantage of better sensitivity to the geophysical parameter but suffers from coarse spatial resolution given limitations on antenna dimensions. Active sensing has the advantage of higher spatial resolution, but the measurements are, relative to radiometry, more affected by the confounding influences of scattering by vegetation and rough surfaces. Active and passive measurements can potentially span different scales and allow the combining of the relative advantages of the two sensing approaches. This strategy is being implemented in the NASA Soil Moisture Active Passive (SMAP) mission, which relies on the relationship between active and passive measurements to provide 9-km surface soil moisture estimates. The aim of this paper is to study the sensitivity of spaceborne L-band active and passive temporal covariations to land surface characteristics, in preparation for SMAP. A significant linear relationship (with slope $\boldsymbol{\beta}$ ) is obtained between NASA's Aquarius scatterometer and radiometer observations across major global biomes. The error in $\beta$ estimation is found to increase with land cover heterogeneity and to be unaffected by vegetation density (up to moderate densities). Results show that $\beta$ estimated with two to eight months of Aquarius measurements (depending on vegetation seasonality) reflect local vegetation cover conditions under surfaces with complex mixture of vegetation, surface roughness, and dielectric constant.
\end{abstract}

Index Terms-Active-passive, Aquarius/SAC-D mission, L-band microwave remote sensing, radiometer, scatterometer, Soil Moisture Active Passive (SMAP) mission.

Manuscript received December 3, 2013; revised June 17, 2014 and December 12, 2014; accepted February 19, 2015

M. Piles and M. Pablos are with the Remote Sensing Laboratory, Departament de Teoria del Senyal i Comunicacions, Universitat Politècnica de Catalunya (UPC), 08034 Barcelona, Spain, and also with IEEC CRAE/ UPC, 08034 Barcelona, Spain. They are also with SMOS Barcelona Expert Center (SMOS-BEC), 08003 Barcelona, Spain (e-mail: maria.piles@ tsc.upc.edu).

K. A. McColl and D. Entekhabi are with the Department of Civil and Environmental Engineering, Massachusetts Institute of Technology, Cambridge, MA 02139 USA.

N. Das is with the Jet Propulsion Laboratory, California Institute of Technology, Pasadena, CA 91109 USA.

Color versions of one or more of the figures in this paper are available online at http://ieeexplore.iee.org.

Digital Object Identifier 10.1109/TGRS.2015.2407611

\section{INTRODUCTION}

$\mathbf{M}$ ICROWAVE remote sensing in the L-band (1-2 GHz) is well suited for the remote sensing of surface soil moisture, representing the state of the land surface water balance. The atmosphere is nearly transparent at these frequencies, and vegetation cover is semitransparent up to moderate densities. Active sensors provide their own source of illumination, containing a transmitter and a receiver, while passive sensors consist only of a receiver that measures the radiation naturally emitted from the scene under observation. Active sensors (radars) are typically capable of remotely sensing surface characteristics at high spatial resolution $(\sim 1 \mathrm{~km}$ or even higher with synthetic aperture processing), but the radar backscatter is highly influenced by combined effects of surface roughness, topography, vegetation canopy structure, and water content [1], [2]. In contrast, passive sensors (radiometers) have a higher sensitivity to soil water content and a reduced sensitivity to land surface roughness and vegetation geometry [3], but their spatial resolution is limited to $\sim 40-50 \mathrm{~km}$ due to practical constraints on antenna size and the altitude of low Earth orbits.

Soil moisture varies on a range of spatial scales due to the combined effects of precipitation, vegetation, soil type, and topography [4]. Accurate observations of soil moisture dynamics are needed to enhance climate prediction skills and weather forecasting [5], which will benefit climate-sensitive socioeconomic activities, including water management, agricultural productivity estimation, flood prediction, and drought monitoring [6], [7]. Two space missions are underway to provide the first dedicated global measurements of the Earth's surface soil moisture using L-band microwave remote sensing: the European Space Agency (ESA) launched the Soil Moisture and Ocean Salinity (SMOS) mission [8] in November 2009, and the National Aeronautics and Space Administration (NASA) plans to launch the Soil Moisture Active Passive (SMAP) mission [9] in January 2015. These two missions each include a radiometer instrument ( $40-\mathrm{km}$ spatial resolution). Additionally, SMAP includes an L-band high-resolution mapping radar onboard, to provide a 9-km soil moisture product by combining active and passive measurements [10], [11].

NASA's Aquarius sensor is a combined active/passive polarimetric L-band microwave instrument launched in 2011, with the primary purpose to provide information on ocean salinity. Although designed for ocean applications, Aquarius is 
providing the first long-term simultaneous L-band active and passive global views of the Earth's surface. Hence, there is a unique opportunity to utilize Aquarius active-passive observations over land to improve our understanding of the geophysical relationship between the radar backscatter and radiometer brightness temperatures. Since scatterometer and radiometer observations offer complementary information on the properties of the observed scene, the combined measurement may be more useful than either one by itself. Temporal variations of combined measurements may provide more effective means to monitor the development of the vegetation canopy and recognize different vegetation types [12]. Analysis of active-passive airborne observations [13] and modeled data [14] indicate that the combination of radar and radiometer could be used to decouple soil effects from vegetation. Thus, they could potentially be used to the benefit of improved soil moisture detection. Also, in a SMAP-like context, an optimal blend of radar and radiometer signatures could lead to high-resolution soil moisture retrievals [15]. The slope of the temporal linear relationship between active and passive observations has been identified as a key parameter for producing the SMAP 9-km product [11].

The primary purpose of this paper is to study the sensitivity of L-band active and passive temporal covariations to land surface characteristics, namely, soil moisture, vegetation density, and landscape cover heterogeneity, in preparation for SMAP. Using a year of Aquarius observations, these covariations are expressed as the slope $\beta$ of the temporal linear relationship between emissivity and backscattering coefficient. Emissivity is used to separate the effect of physical temperature variations on brightness temperatures. The Radar Vegetation Index (RVI) [16] is used as an indicator of canopy cover. The Gini-Simpson index (GSI) [17] is employed to assess the impact of heterogeneity of surface characteristics within a satellite footprint. The results of this study are relevant to the design of multiresolution active-passive surface soil moisture retrievals in general. Although Aquarius radiometer and scatterometer use the same aperture and hence have comparable resolutions, radar systems can potentially provide higher resolution measurements using synthetic aperture processing. This approach is being implemented in the SMAP mission. The SMAP baseline multiresolution active-passive retrieval relies on the estimation of parameter $\beta$ to provide intermediate (to radar and radiometer) surface soil moisture estimates. Although the Aquarius-based estimates of this parameter are at far coarser and incompatible resolutions than those possible and required with SMAP, the results of this study provide some fundamental insights into the role of landscape vegetation cover on the seasonal and geographical distribution of the L-band active and passive measurement covariability.

\section{Physics of Microwave Remote SENSING OVER LAND}

This section provides an overview of the theoretical background to active and passive microwave remote sensing over land surfaces. A more comprehensive review can be found in [3].

\section{A. Random Surface Scattering and Emission}

Wave scattering and emission from random rough surfaces are characterized by the bistatic scattering cross section per unit area or surface scattering coefficient $\left(\sigma^{0}\right)$. It relates the magnitude of the power scattered in the direction $\left(\theta_{s}, \phi_{s}\right)$ with polarization $q$ to the power incident in the surface from the direction $\left(\theta_{0}, \phi_{0}\right)$ with polarization $p[3]$. Applying Kirchoff's radiation law, Peake [18] demonstrated that polarized emissivity $e_{p}$ in a chosen observation direction is equal to one minus reflectivity $\Gamma_{p}$, expressed as the integral value of the bistatic scattering cross section over the upper half space

$$
\begin{aligned}
e_{p ; \theta} & =1-\Gamma_{p ; \theta} . \\
\Gamma_{p ; \theta_{0}, \phi_{0}} & =\frac{1}{4 \pi \cos \theta_{0}} \iint\left[\sigma_{p p ; \theta_{0}, \phi_{0} ; \theta_{s}, \phi_{s}}^{0}+\sigma_{p q ; \theta_{0}, \phi_{0} ; \theta_{s}, \phi_{s}}^{0}\right] d \Omega_{s},
\end{aligned}
$$

where $d \Omega_{s}=\sin \theta_{s} d \phi_{s} d \theta_{s}$ and $\theta$ and $\phi$ are the incidence and azimuth angles, respectively.

Scattering by a rough surface presents simple analytical solutions for a limited number of cases only, which correspond to some combinations of roughness parameters and observation frequencies. The traditional models include the small perturbation method (SPM) and the Kirchhoff approximation (KA) [19]. The SPM is valid for slightly rough surfaces, while the KA is applicable for a rough surface with a large surface curvature. The integral equation model overlaps these two methods and has been proven to have a wider range of applicability than the classical methods [20]. Validation of the approximate methods has been performed by exact numerical methods [21] or experimental data, mostly collected on conducting surfaces [20]. Validation using remotely sensed data over natural terrains is complex, since the bistatic scattering coefficient measured at various incidence and scattering angles on the whole upper space would be needed to validate model performances. However, radars measure the backscattering coefficient, which is a particular case of $\sigma^{0}$ for the incidence and scattered directions of the emitting and receiving antennas. In turn, radiometers measure brightness temperatures-directly related to emissivity - within a limited range of incidence angles.

In this paper, the relationship between emissivity and radar backscatter will be estimated statistically using Aquarius passive measurements globally, for surfaces with a complex mixture of vegetation and surface roughness characteristics.

\section{B. Surface Emissivity}

In the microwave region, the dielectric constant of most natural materials is dominated by its water content. The dielectric constant is a measure of the surface response to an electromagnetic wave; it is defined as a complex number $\left(\epsilon_{s}=\epsilon_{s}^{\prime}+j \epsilon_{s}^{\prime \prime}\right)$, where the real part determines the propagation characteristics of the energy as it passes through the surface and the imaginary part determines the energy losses. The theory behind passive L-band microwave remote sensing is based on the large contrast between the dielectric constant of dry soil ( $\sim 4)$ and water $(\sim 80)$. This contrast results in a broad range of dielectric 
values for soil-water mixtures $\left(\epsilon_{s} \approx 4-40\right)$ and is the primary influence on the natural microwave emission from the soil [22].

The thermal radiation or brightness temperature emitted by the Earth's surface $\left(T_{B_{p, \theta}}\right)$ for bare soil is determined by its physical temperature $T_{p h}$ and its emissivity $e_{p, \theta}$, according to

$$
T_{B_{p, \theta}}=e_{p, \theta} \cdot T_{p h},
$$

where the subscript $p$ denotes either vertical $(V)$ or horizontal $(H)$ polarization and $\theta$ is the incidence angle.

The $\tau-\omega$ model [23] is generally used to account for the effects of vegetation on the observed microwave signal

$$
\begin{aligned}
T_{B_{p, \theta}}=e_{p, \theta} T_{s} e^{-\tau / \cos \theta}+(1-\omega) T_{v}\left(1-e^{-\tau / \cos \theta}\right) \\
+\Gamma_{p, \theta}(1-\omega) T_{v}\left(1-e^{-\tau / \cos \theta}\right) e^{-\tau / \cos \theta},
\end{aligned}
$$

where $T_{s}$ and $T_{v}$ are the effective temperatures of the soil and the vegetation, respectively, $\tau$ is the vegetation opacity, and $\omega$ is the vegetation single-scattering albedo. The first term of the aforementioned equation defines the radiation from the soil as attenuated by the overlying vegetation. The second term accounts for the upward radiation directly from the vegetation, while the third term defines the downward radiation from the vegetation, reflected upward by the soil and again attenuated by the canopy.

At Aquarius morning overpasses (6 A.M.), temperature gradients within the soil and vegetation are minimized so that the temperature of the vegetation canopy is in equilibrium with the soil temperature $\left(T_{s}=T_{v}=T_{p h}\right)$ [24]. Also, under low vegetation cover conditions, the single-scattering albedo can be neglected since $\omega \ll 1$. Under these assumptions and using (1), (4) becomes simply

$$
T_{B_{p, \theta}}=T_{p h}\left(1-\Gamma_{p, \theta} e^{-2 \tau / \cos \theta}\right) .
$$

Surface reflectivity can be decomposed as $\Gamma_{p, \theta}=\Gamma_{p, \theta}^{*} e^{-h}$, where $h$ is the roughness parameter related to the root mean square of surface roughness and $\Gamma_{p, \theta}^{*}$ stands for the power reflection of the flat soil. Now, (5) becomes

$$
T_{B_{p, \theta}}=T_{p h}\left(1-\Gamma_{p, \theta}^{*} e^{-h} e^{-2 \tau / \cos \theta}\right) .
$$

Using the Fresnel equations, $\Gamma_{p, \theta}^{*}$ can be related to soil moisture through the dielectric constant.

\section{Surface and Volume Scattering}

Terrain scattering is governed by both the surface roughness and its dielectric properties. If the roughness of a surface remains unchanged, the measured radar backscattering increases with increasing moisture content and may exhibit a dynamic range of about $10 \mathrm{~dB}$ between dry soil and very wet soil conditions [25].

Electromagnetically, a surface is considered smooth if its height variations are much smaller than the wavelength of the radiation. Scattering from a perfectly flat surface consists of only the specularly reflected coherent part, which is given by a Dirac delta function centered at the specular direction. Scattering from a slightly rough surface consists of a large reflected coherent component in the specular direction and a small noncoherent or diffuse scattered component which consists of power scattered in all directions. As the surface becomes rougher, the coherent component becomes negligible, and the scattering pattern is composed entirely of the diffuse component. For the perfectly rough surface, emissivity (and reflectivity) is polarization and angle independent [3].

When the soil is covered by vegetation, the backscattering coefficient at polarization $p q(=H H, V V$, or $H V)$ and incidence angle $\theta$ is highly influenced by the canopy structure, its biomass, and its water content and can be described as a sum of three dominant contributions

$$
\sigma_{p q, \theta}^{0}=\sigma_{p q, \theta}^{s} e^{-2 \tau / \cos \theta}+\sigma_{p q, \theta}^{v}+\sigma_{p q, \theta}^{s v} .
$$

The first term represents the scattering cross section of the soil surface $\sigma_{p q, \theta}^{s}$, a function of $\epsilon_{s}$ and soil roughness characteristics, which is modified by the two-way vegetation attenuation $\left(e^{-2 \tau / \cos \theta}\right)$. The second term $\sigma_{p q, \theta}^{v}$ is the scattering cross section of the vegetation volume, an intricate function of vegetation alone. The third term $\sigma_{p q, \theta}^{s v}$ represents the scattering interaction between the soil and vegetation, which depends on $\epsilon_{s}$, soil roughness characteristics, and vegetation canopy in complex ways [3].

Surface scattering is dominated by like-polarized backscatters $\left(\sigma_{V V}\right.$ and $\left.\sigma_{H H}\right)$, whereas cross-polarized backscatters $\left(\sigma_{H V}=\sigma_{V H}\right)$ are typically associated with volume or multiple scattering. This is due to the differing depolarization effects associated with these mechanisms. Surface scattering does not cause a significant change in polarization, so the crosspolarized receiving antenna receives little energy from bare soil or rock. For vegetated areas, a significant depolarization of the radar signal occurs: it penetrates to varying degrees (depending on wavelength) and interacts with stems, leaves, twigs, and trees' branches, leading to scattered waves vibrating in various directions.

\section{DAta AND Methods}

\section{A. Aquarius Data}

The Aquarius/SAC-D mission, launched in June 2011, is a joint U.S./Argentinian mission to map the surface salinity field of the oceans from space. It has equatorial crossing times of 6 A.M. (descending) and 6 P.M. (ascending) and a sevenday repeat cycle. Its primary payload is the NASA Aquarius sensor, the first combined active/passive polarimetric L-band microwave instrument in space. It consists of three radiometers at $1.413 \mathrm{GHz}$ and a scatterometer (a real aperture radar) at $1.26 \mathrm{GHz}$, which sequences with the three radiometer' antenna beams so as to have active/passive collocated observations [26]. The spacecraft orbits the Earth with the sun at the left and the three beams pointing across-track toward the right, arranged to image in a pushbroom fashion with $29.36^{\circ}$ (inner beam), $38.49^{\circ}$ (middle beam), and $46.29^{\circ}$ (outer beam) incidence angles, with footprints of $76 \times 94 \mathrm{~km}, 84 \times 120 \mathrm{~km}$, and $96 \times 156 \mathrm{~km}$, respectively, providing a swath of about $390 \mathrm{~km}$. 


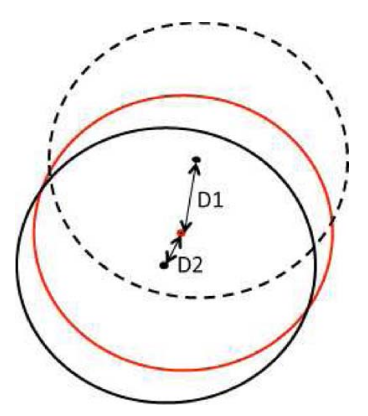

Fig. 1. Schematic of the sampling method used to grid Aquarius data. Footprints with centers less than a threshold $\left(0.22^{\circ}\right.$ in this study) are included in the grid cell (red). Here, D2 $<0.22$, so the corresponding footprint is included in the grid cell (solid black); D1 $>0.22$, so the corresponding footprint is excluded (dashed black).

In the present study, version 2.0 Aquarius brightness temperature ( $H$ and $V$ polarizations) and normalized radar cross section ( $H H, V V$, and $H V$ polarizations) data from the descending overpasses at $38.49^{\circ}$ incidence angle (central beam) have been used. Descending overpasses are preferred to minimize the gradients between the soil and vegetation temperature (see Section II). Data from the central beam are selected since, out of the three available beams, more independent information can be obtained from the vertical and horizontally polarized brightness temperatures at $38.49^{\circ}$; it is also the closest to SMAP constant incidence angle $\left(40^{\circ}\right)$. The data have been screened for all Aquarius maneuvers and radio frequency interference in either the radar or radiometer. In addition, data over ocean, landsea transitions, Antarctica, and Greenland have been masked out. One year of worldwide observations has been considered, from September 1, 2011, to August 31, 2012. This is the full annual cycle of Aquarius measurements available at the time of this study.

Land surface temperature and soil moisture data from the National Centers for Environmental Prediction (NCEP) are provided along with Aquarius observations as auxiliary information. They are interpolated from the daily $0.25^{\circ}$ product to the exact time and location of the Aquarius observations. In this paper, Aquarius brightness temperatures $T_{B_{p, \theta}}$ are normalized by NCEP collocated land surface temperature using (3) to eliminate dependences between $T_{p h}$ and $T_{B_{p, \theta}}$.

\section{B. Footprint Gridding}

Since Aquarius measurements do not exactly overlap across half-orbits over time, a sampling strategy needs to be defined in order to produce global temporal views. The first seven days of observations have been used to set up a grid (see the red circle on Fig. 1). Subsequent overlapping footprints with centers less than $0.22^{\circ}$ from a grid center are included in that grid cell (e.g., black circle, Fig. 1). Otherwise, they are excluded (e.g., dashed circle, Fig. 1). Note that some observations may be included in multiple grid footprints. This gridding strategy has been chosen so as to keep the spatial resolution of the analysis to the instrument's spatial resolution.

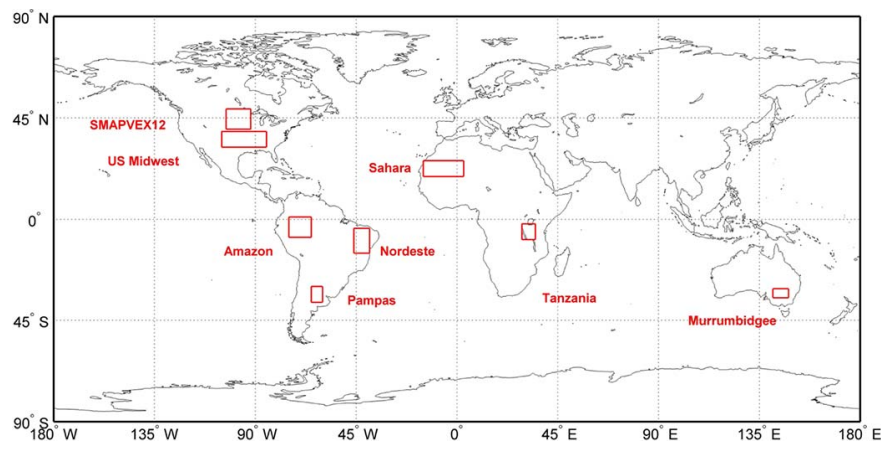

Fig. 2. Red squares delineate the location and extent of target areas.

\section{IGBP Land Cover}

The 2005 MODIS MCD12Q1 International GeosphereBiosphere Program (IGBP [3]) collection 5 land cover product [4] has been used in this study to characterize the dominant land cover within the target areas and to account for surface heterogeneity. It is a worldwide product at $500-\mathrm{m}$ spatial resolution that encloses 17 distinctive land cover classes. In this paper, the dominant IGBP land cover type has been determined for each Aquarius footprint. MODIS products are freely distributed by the U.S. Land Processed Distributed Active Archive Center (www.lpdaac.usgs.gov).

\section{Target Areas}

Due to the poor dynamic range of the data on a global basis, eight target areas were selected for more detailed study of temporal behaviors, vegetation, and heterogeneity impacts. These target areas are shown in Fig. 2. They were selected to be representative of a wide range of vegetation characteristics and heterogeneity within a $\sim 100-\mathrm{km}$ Aquarius footprint across major biomes. Also, they are among the SMAP calibration/validation core sites so that the experience gained about them is useful for broader applications; core sites such as the Murrumbidgee catchment [27], SMAPVEX12 [28], and U.S. Midwest [29] are equipped with soil moisture networks that routinely acquire ground data. Specific location, area, dominant land cover type, median RVI (a measure of vegetation scattering; see Section IV-C), heterogeneity (given as median GSI; see Section IV-D), and number of Aquarius collocated active-passive observations acquired at each target area are included in Table I. Note that the areas in Table I are ordered by increasing canopy cover according to IGBP, where Sahara and Amazon are end members. For the target area analysis, all of the Aquarius measurements with footprints entirely enclosed within the target areas' boundaries were selected. No gridding was performed.

\section{Relationship Between Aquarius ACTIVE AND PASSIVE OBSERVATION}

As indicated in Section II, both surface emissivity and backscatter cross section at L-Band respond to changes in surface soil dielectric constant. Hence, they are both indicators of surface soil moisture volumetric content. However, the two 
This article has been accepted for inclusion in a future issue of this journal. Content is final as presented, with the exception of pagination.

TABLE I

DESCRIPTION OF TARGET AREAS

\begin{tabular}{lcclccc}
\hline Site name & \multicolumn{2}{c}{ Location } & Area $\left(\mathrm{km}^{2}\right)$ & Dominant IGBP & \multicolumn{2}{c}{ Median RVI Median GSI Number of observations } \\
\hline Sahara & $15^{\circ} \mathrm{W}: 3^{\circ} \mathrm{E}-19^{\circ} \mathrm{N}: 26^{\circ} \mathrm{N}$ & $1438 \cdot 10^{3}$ & Barren & 0.12 & 0 & 14677 \\
Pampas & $65^{\circ} \mathrm{W}: 60^{\circ} \mathrm{W}-37^{\circ} \mathrm{S}: 30^{\circ} \mathrm{S}$ & $361 \cdot 10^{3}$ & Cropland & 0.39 & 0.48 & 4428 \\
SMAPVEX12 & $103^{\circ} \mathrm{W}: 92^{\circ} \mathrm{W}-40^{\circ} \mathrm{N}: 49^{\circ} \mathrm{N}$ & $872 \cdot 10^{3}$ & Cropland & 0.38 & 0.59 & 4328 \\
US Midwest & $105^{\circ} \mathrm{W}: 85^{\circ} \mathrm{W}-32^{\circ} \mathrm{N}: 39^{\circ} \mathrm{N}$ & $1408 \cdot 10^{3}$ & Grassland & 0.65 & 0.79 & 3585 \\
Murrumbidgee & $141^{\circ} \mathrm{E}: 148^{\circ} \mathrm{E}-35^{\circ} \mathrm{S}: 31^{\circ} \mathrm{S}$ & $290 \cdot 10^{3}$ & Open Shrubland & 0.69 & 0.43 & 3732 \\
Tanzania & $29^{\circ} \mathrm{E}: 35^{\circ} \mathrm{E}-9^{\circ} \mathrm{S}: 2^{\circ} \mathrm{S}$ & $516 \cdot 10^{3}$ & Savanna & 0.80 & 0.72 & 3549 \\
Nordeste & $46^{\circ} \mathrm{W}: 39^{\circ} \mathrm{W}-15^{\circ} \mathrm{S}: 4^{\circ} \mathrm{S}$ & $937 \cdot 10^{3}$ & Woody Savanna & 0.87 & 0.35 & 9841 \\
Amazon & $75^{\circ} \mathrm{W}: 65^{\circ} \mathrm{W}-8^{\circ} \mathrm{S}: 1^{\circ} \mathrm{N}$ & $1109 \cdot 10^{3}$ & Evergreen Broadleaf Forest & 1.00 & 0.04 & 13061 \\
\hline
\end{tabular}

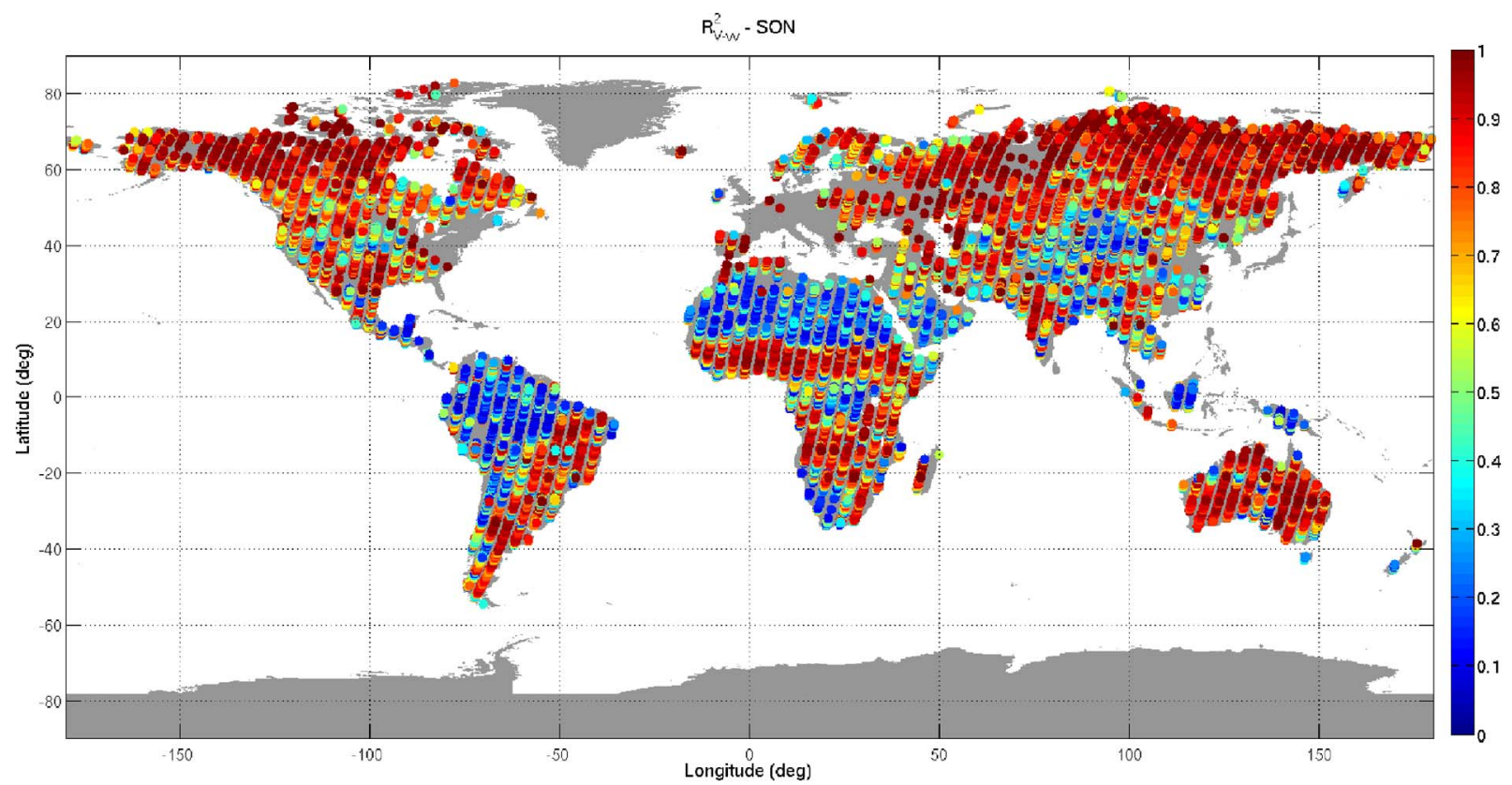

Fig. 3. Percentage explained variance $\left(R^{2}\right)$ between passive $e_{V}$ and active $\sigma_{V V}$ Aquarius measurements from September-October-November 2011.

measurements are variably affected by the surface roughness and vegetation canopy characteristics. These differences are potentially a major obstacle to the joint use of the two measurements for more robust geophysical retrievals. On the other hand, the differences can be regarded as an opportunity for enhanced science data products, especially if they are characterized by different resolutions. This paper studies the synergy of Aquarius active and passive observations in pursuit of those opportunities.

\section{A. Strength of Relationship}

To study the temporal covariability between Aquarius passive $e_{p}$ and active $\sigma_{p q}$ observations over land, the percentage variance explained by linear regression $R^{2}$ was first calculated. Fig. 3 shows $e_{V}-\sigma_{V V} R^{2}$ for a period of three months (from September 1 to November 30, 2011). It demonstrates the strength of the active-passive relationship in Aquarius measurements. Note that exceptions $\left(R^{2}<0.7\right)$ are in densely vegetated areas such as the Amazon and the Congo, where the L-band radar and radiometer cannot penetrate, and in areas where there are no dynamics in soil moisture (e.g., deserts). Also, some coastal areas present low values of $R^{2}$, which could be an effect of land-sea contamination. This analysis has also been performed using the other possible polarization combinations $e_{V}-\sigma_{H H}, e_{H}-\sigma_{V V}$, and $e_{H}-\sigma_{H H}$ (not shown) with comparable results. The $e_{V}-\sigma_{V V}$ combination is shown here as it had the highest degree of correlation in airborne observations from SGP99, SMEX02, CLASIC, and SMAPVEX08 field experiments [10, Fig. 2] and is the one reported in the follow-on studies [11].

\section{B. Temporal Linear Relationship}

Since Aquarius makes coincident and constant look-angle radar and radiometer measurements, their covariations over short time periods can be considered mostly related to changes in soil dielectric constant - that depends on surface soil moisture changes-rather than contributions of vegetation and surface roughness. The latter two factors generally change over long time periods such as seasons: surface roughness changes mostly due to disturbances, erosion, and deposition events; vegetation characteristics follow seasonal phenology. Agricultural practices such as harvesting and ploughing, however, may markedly change vegetation and surface roughness, respectively, within short periods of time. 


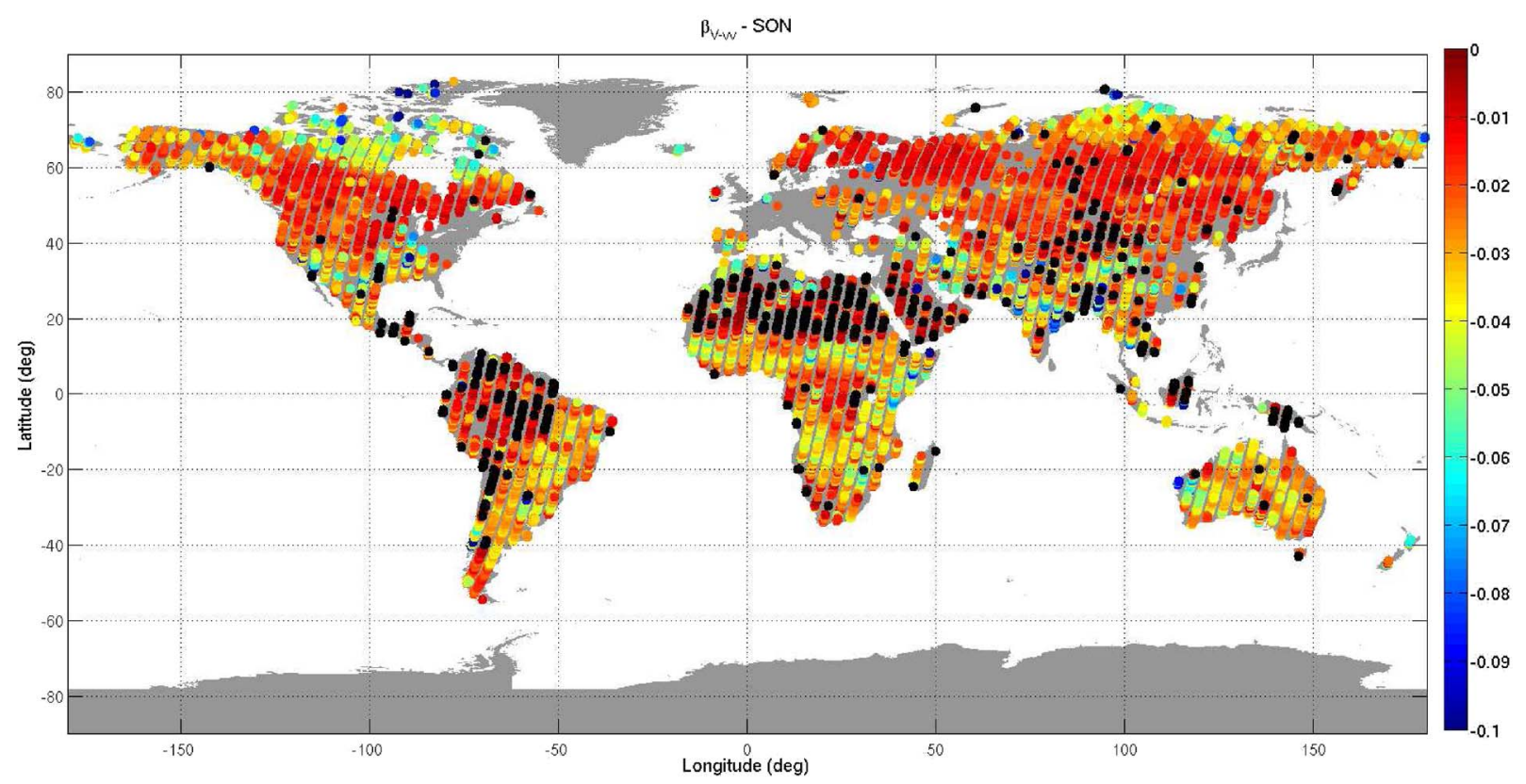

Fig. 4. Parameter linking active and passive measurements $(\beta)$ : slope of $e_{V}-\sigma_{V V}$ linear relationship obtained using temporal series of Aquarius data from September-October-November 2011. Only values with statistical significance $(p<0.05)$ are shown with color shading. Black dots indicate positive values, outside the valid range of $\beta$.

Taking advantage of the approximately linear temporal relationship between radar backscatter and brightness temperature, the slope $\beta$ of the linear regression $e_{V}=\alpha+\beta \cdot \sigma_{V V}$ is estimated for each grid cell using three months of data (from September 1 to November 30, 2011). The resulting global fields of $\beta$ are filtered by statistical significance. Only values with statistical significance $(p<0.05)$ are shown as color-shaded pixels in Fig. 4.

According to theory, the slope of the linear regression between emissivity and backscatter under $50^{\circ}$ incidence angles - which is the case of Aquarius and upcoming SMAP observations-is positive for water surfaces (high dielectric constant). Land surfaces with low dielectric constant (low soil moisture) will typically have a negative slope, and this slope may change from negative to positive when the dielectric constant increases (due to a soil moisture increase or complex surface roughness effects) [3]. Over three months, changes in soil moisture state are expected to dominate the active-passive temporal covariability, with an increase of surface soil moisture leading to a decrease in emissivity (at polarization $v$ or $h$ ) and an increase in radar co-pol ( $h h$ or $v v$ ) measurements, and viceversa. In this scenario, soil moisture variations in time result in TB and $\sigma_{p p}$ being negatively correlated. As expected, Fig. 4 shows that Aquarius three-month $\beta$ estimates over landmasses are generally negative, with positive values (in black) mostly located in dense forests, where L-band does not penetrate, areas with no dynamics in soil moisture (deserts), and coastal areas. The statistical estimation of $\beta$ is susceptible to noise as evident in the random but sparse occurrence of positive values away from dense forests, deserts, and coastal regions.

Recent studies on the linear functional relationship between time series of $T_{B}$ and $\sigma_{p}$ have been performed using airborne passive and active data from multiple field campaigns in [30]. They show the strength of the $T_{B}-\sigma_{p p}$ linear relationship and report that the slope changes over time are negative in sign and its magnitude decreases for denser vegetation conditions. In this paper, $\beta$ is estimated from Aquarius emissivity and radar backscatter, which prevents direct comparison of magnitudes in kelvin per decibel reported in the literature. However, this study demonstrates that $\beta$ (qualitative) estimates from a satellite platform are in line with results from experimental campaigns. Over a three-month period, Fig. 4 shows that global $\beta$ estimates are statistically robust and correspond with expected land cover characteristics. It is foreseen that $\beta$ varies seasonally as well as geographically. Its seasonal dependence is examined in Section IV-E, where the impact of the temporal window used in $\beta$ estimation is discussed.

Scatter plots of Aquarius passive $e_{V}$ against active $\sigma_{V V}$ observations acquired over the target areas are presented in Fig. 5. Plots are shown for the hemisphere warm season (three months) at each target area. These are representative of the remaining seasons at the target areas (not shown) with expected but only slight variations in the slope and dynamic range of the data. Seasonality in soil moisture and vegetation canopy are reflected in the plots. The variation in estimated $\beta$ per target area across time periods demonstrates its dependence on roughness and seasonally varying vegetation phenology (this is further discussed in Section IV-C). The range of emissivity in the $y$-axis is common for all of the plots, and the range of radar backscatter in the $x$-axis is set to $14 \mathrm{~dB}$ for all target areas except for Sahara, where it is set to $24 \mathrm{~dB}$. This is needed to make the plots comparable and, at the same time, show full $\sigma_{V V}$ variability. Colors indicate the NCEP volumetric soil moisture associated with each pair of collocated observations. A soil moisture gradient can be observed in most areas from dry soils (high $e_{V}$ and low $\sigma_{V V}$, in red) to wet soils (low $e_{V}$ and high $\sigma_{V V}$, in blue), which reflects the inverse relationship of Aquarius active and passive observations with soil dielectric constant, and a general agreement with NCEP soil moisture estimates. 

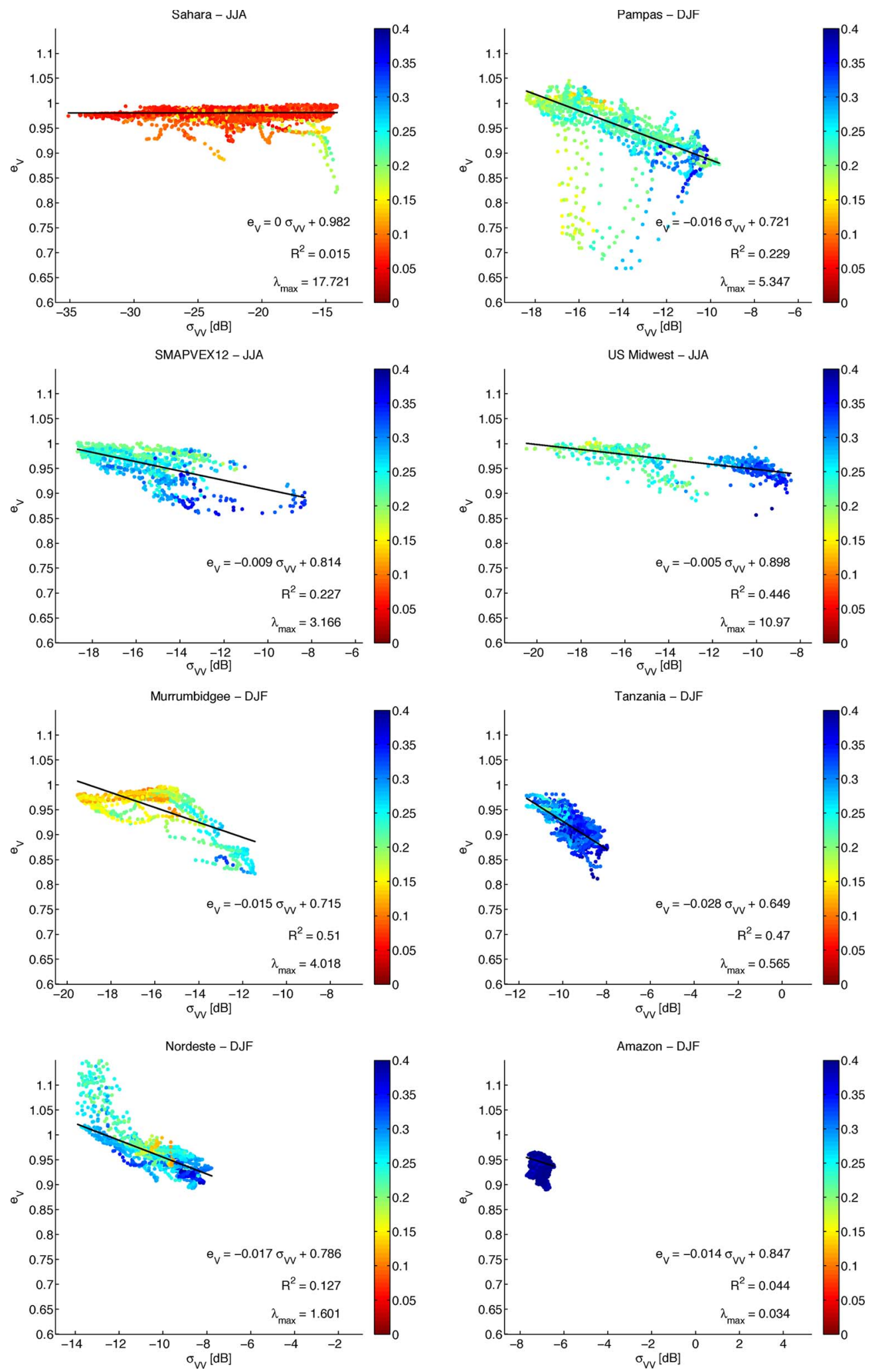

Fig. 5. Scatter plots of Aquarius radiometer $e_{V}$ versus radar $\sigma_{V V}$ over the target areas on Fig. 2. Hemisphere warm season (three months) plots are shown for each focus area. Other seasons (not shown) show similar regional behavior with slight changes in slope and dynamic range of data consistent with climate seasonality. Colors indicate the NCEP soil moisture $\left[\mathrm{m}^{3} / \mathrm{m}^{3}\right]$ associated to each pair of collocated observations. The variance $\lambda_{\text {max }}$ explained by the principal component of the scatter plots is used as an indicator of the dynamic range and the spread of the observations. Nonphysical emissivity values $\left(e_{V}>1\right)$ can be explained by inconsistencies between NCEP land surface temperatures and Aquarius brightness temperatures.

Fig. 5 shows that the linear relationship between $e_{V}-\sigma_{V V}$ holds in all of the studied regions except for Sahara and Amazon, as expected. In the Amazon, dense vegetation domi- nates the apparent surface emissivity and backscatter, with both active and passive measurements having a low dynamic range and not following even a seasonal cycle. The Tanzania region 
exhibits a low dynamic range (more than the Amazon but still lower than other regions) for the emissivity and backscattering coefficient, possibly due to heavy vegetation cover. Also, this region comprises several inland water bodies, which could lead to the reduced dynamic range in emissivity. Both of these factors result in a large value of $\beta(-0.028)$. In the Sahara, which is a desert, the soil moisture is consistently low throughout the year. The red color of the data in the Sahara panel of Fig. 5 is due to persistent dessicated surfaces. As a result of the lack of dynamic range in the emissivity, the slope between $e_{V}-\sigma_{V V}$ is near zero. During the few precipitation episodes that moisten the surface, even the Sahara data show a sloped relationship between emissivity and radar backscatter cross section. Over the U.S. Midwest, South America Pampas, and Nordeste regions, a clear (negative slope) relationship between emissivity and backscatter coefficient is evident. This is also evident across the Murrumbidgee basin where SMAPEx airborne experiments are conducted [31]. However, not enough coincident airborne active and passive measurements are available to independently estimate $\beta$. Over SMAPVEX, a $\beta$ value of -0.009 is obtained using three months of Aquarius data. During the Soil Moisture Active Passive Validation Experiment 2012 (SMAPVEX12) in June-July 2012, an average $\beta$ value of -0.018 is obtained using nearly a month of Passive Active L-band System (PALS) airborne data. However, the comparison of the obtained magnitudes is subject to time- and spatial-sampling differences. Regarding $\beta$ estimation time, there are not enough Aquarius overpasses to match PALS estimation more precisely in time, so the $\beta$ value is obtained using nearly one-month period for PALS and using three months for Aquarius. Regarding data spatial coverage, the SMAPVEX12 domain covered by PALS is nearly $2500 \mathrm{~km}^{2}$, whereas the Aquarius footprint size is $10000 \mathrm{~km}^{2}$. Also, $T_{B}$ footprints impacted by water bodies and urban areas are removed from PALS data and not from Aquarius; the presence of water bodies, urban areas, and forest in the larger footprint of Aquarius could explain the reduced magnitude of $\beta$.

Heterogeneity in surface parameters, especially vegetation cover, is suspected to affect the $e_{V}-\sigma_{V V}$ linear relationship. In Section IV-D, an index of vegetation heterogeneity is introduced to analyze the impact of land surface heterogeneity on $\beta$ estimation.

\section{Impact of Canopy Cover}

In this paper, the RVI [16] is used as a measure of vegetation scattering. It is a polarimetric parameter defined by

$$
\mathrm{RVI}=\frac{8 \cdot \sigma_{H V}}{\sigma_{H H}+\sigma_{V V}+2 \cdot \sigma_{H V}}
$$

where the radar backscatter cross sections are in units of power. RVI should vary between zero for nearly bare soil $\left[\sigma_{H V} \ll\right.$ $\left(\sigma_{H H}\right.$ or $\left.\left.\sigma_{V V}\right)\right]$ and unity for full volume scattering by dense vegetation $\left[\left(\sigma_{H H}\right.\right.$ and $\left.\left.\sigma_{V V}\right) \approx 3 \cdot \sigma_{H V}\right]$. Since Aquarius $\sigma_{H V}$ is not well calibrated over land, RVI will only be used as an indicator of canopy cover. A thorough RVI error analysis using Aquarius scatterometer observations can be found in [32].
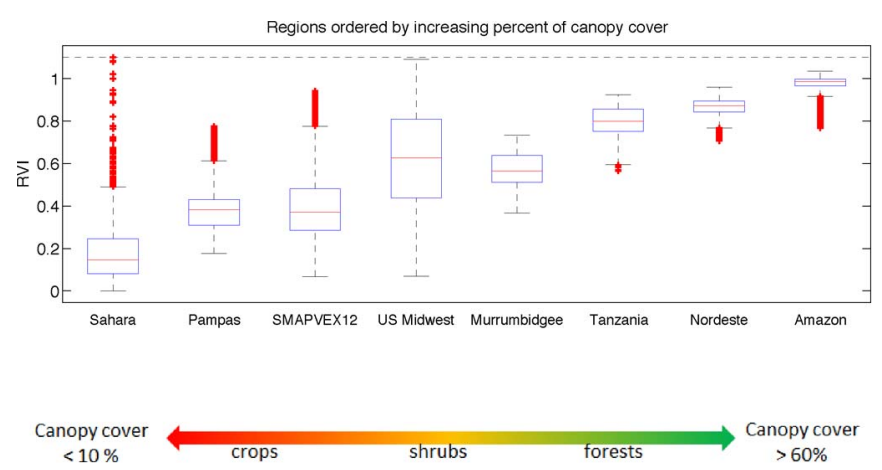

Fig. 6. Boxplot of RVI versus target areas ordered by increasing canopy cover, according to its dominant IGBP land cover.

A boxplot containing RVI statistics over a year-long Aquarius radar data at the target areas is shown in Fig. 6. The target areas in the $x$-axis have been ordered from left to right by increasing canopy cover, according to its dominant IGBP land cover. A clear correlation of RVI with percentage of canopy cover can be observed, confirming the adequacy of its use as a measure of vegetation scattering. Note that croplands (Pampas and SMAPVEX12) and agricultural regions (U.S. Midwest) exhibit a higher RVI variance, which is consistent with their changing (seasonal) vegetation patterns.

The scatter plots in Fig. 5 show that the dynamic range of the passive and active observations is significantly reduced with increasing canopy cover, especially in forested areas, i.e., Tanzania, Nordeste, and Amazon. As expected, the presence of a vegetation canopy reduces the dynamic range of radar backscatter cross section faster than that of the radiometer brightness temperatures. The Sahara region requires particular attention. Radiometric observations over the Sahara have a very limited range, whereas the radar observations extend across all its dynamic range $(\sim 20 \mathrm{~dB})$. The limited range of $e_{V}$ can be explained by the absence of precipitation and vegetation changes in this region. The wide range of $\sigma_{V V}$ can, in turn, be explained by the strong winds producing changes in surface roughness. This example illustrates that passive sensors have a reduced sensitivity to land surface roughness, and in contrast, active observations are highly influenced by it.

To further analyze the change of dynamic range of passive and active observations with percentage of canopy cover, a principal component analysis has been performed with the observations acquired over each target area during the study period. The variance $\lambda_{\max }$ explained by the principal component of the scatter plot $e_{V}-\sigma_{V V}$ at every target area has been computed as an indicator of the dynamic range and the spread of the observations (see annotations on Fig. 5). Fig. 7 shows $\lambda_{\max }$ versus the median RVI observed at the target areas. The decrease of $\lambda_{\max }$ with increasing RVI is clear in all target areas except for Sahara and U.S. Midwest. As already discussed, the high $\lambda_{\max }$ in the Sahara is due to wind inducing surface roughness changes. The high $\lambda_{\max }$ in the U.S. Midwest could be mainly due to its high heterogeneity $(\mathrm{GSI}=0.79)$. Also, a strong change in surface roughness or vegetation conditions may have significantly affected radar observations over the area. Note that its $\sigma_{V V}$ is the second greatest, after Sahara. 


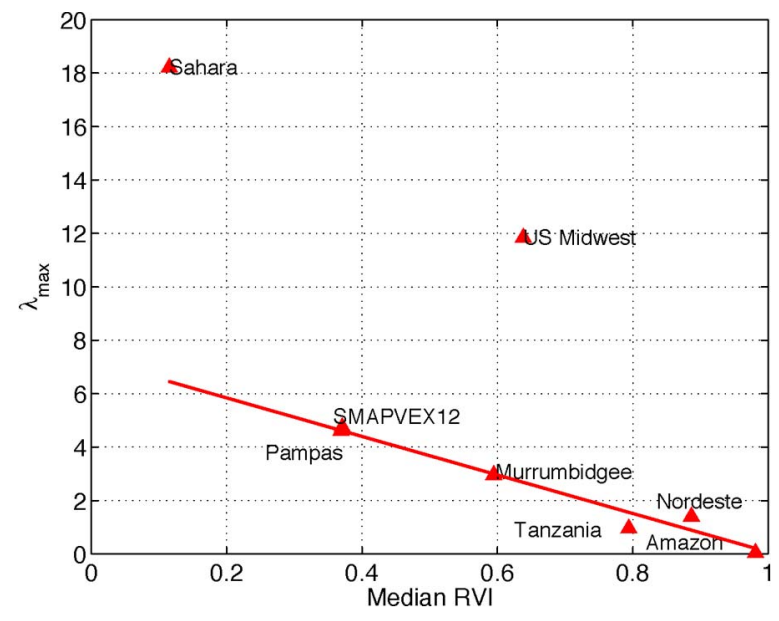

Fig. 7. Plot of the variance explained by the principal component of the scatter plot $e_{V}-\sigma_{V V}\left(\lambda_{\max }\right)$ versus median RVI at target areas. Sahara and Amazon have not been included in the regression.

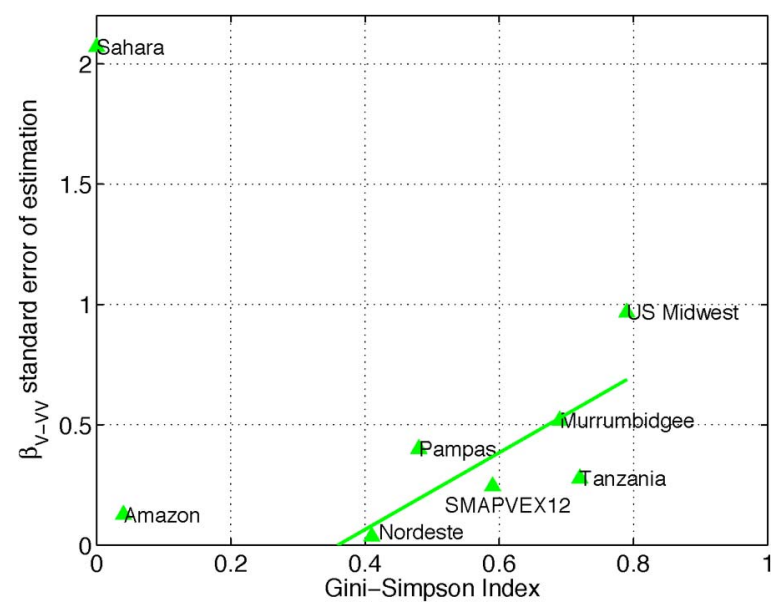

Fig. 8. Plot of $\beta$ standard error of estimation versus median GSI at target areas. Sahara and Amazon have not been included in the regression.

\section{Impact of Heterogeneity}

In this paper, we propose the use of the GSI as a measure of land cover heterogeneity. Based on the work of [17] and [33], GSI is a common measure of diversity that is equal to the probability that two values drawn from a dataset, with replacement, will be of different types. It is defined as

$$
\mathrm{GSI}=1-\sum_{i} p_{i}^{2}
$$

where $p_{i}$ is the proportion of pixels belonging to IGBP class $i$. GSI always lies within the interval $[0,1)$, with its value approaching unity as land class diversity becomes large (no matter how dissimilar the communities are [34]).

Accounting for land heterogeneity is critical in the retrieval of geophysical parameters from satellite observations since it can significantly degrade the quality of the retrievals. In this paper, the median GSI of each target area has been calculated (see Table I) to account for their heterogeneity and is used to assess the limitations imposed by heterogeneity of vegetation cover and soil characteristics within a satellite footprint. Fig. 8 displays the median GSI of each target area against $\beta$ standard

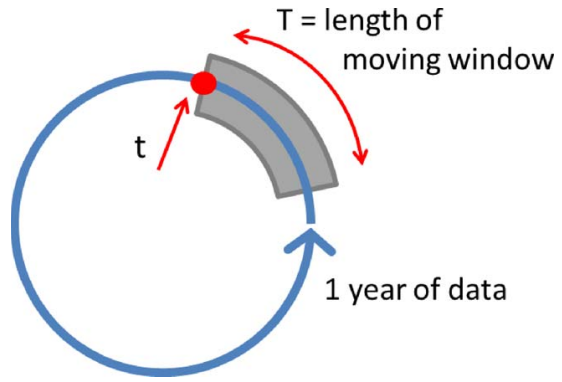

Fig. 9. Schematic of circular moving window used to obtain a set of $\beta$ estimations with one year of Aquarius data.

error of estimation. Excluding the Sahara and Amazon as being extreme cases with no changes in soil moisture and having dense forest, respectively, it shows that the error on $\beta$ estimates increases linearly with the degree of heterogeneity. The error on $\beta$ estimates over the U.S. Midwest is probably increased due to rapidly changing crop conditions (growth and harvest) during the study period. This result indicates that heterogeneity in vegetation may introduce a bias in the estimation of $\beta$. This may be more problematic for instruments with coarse footprint data such as Aquarius and generally in regions with strong vegetation gradients.

\section{E. Impact of Temporal Window}

As discussed in the aforementioned sections, the statistically estimated slope $\beta$ is specific for a given location and reflects the fixed local roughness and vegetation cover conditions. This section addresses $\beta$ dependence on the temporal window used to estimate it. If the moving window is too long, vegetation properties will change with season, and the linear relationship between emissivity and backscatter will be weakened, compromising $\beta$ estimation. Conversely, if the window is too short, sample sizes will be too small, and $\beta$ estimates will be statistically insignificant. In addition, $\beta$ estimation is possible only if there is adequate soil moisture variation within the window period (drydown or wetting event) to cause variations in both $T_{B}$ and $\sigma_{p p}$ to allow estimation of a slope in the regression.

A circular moving window (see Fig. 9) has been defined to assess how long the radar-radiometer time series needs to be for a robust estimate of $\beta$. Regression of the time series (formed based on multiple overpasses, no gridding or averaging) for $e_{V}$ and $\sigma_{v v}$ is used to statistically estimate $\beta$ for each target area using moving window lengths $T$ of one month, three months, six months, and one year, with $t$ time step of one week. Results are shown in Fig. 10 for Pampas, SMAPVEX12, U.S. Midwest, Murrumbidgee, Tanzania, and Nordeste target areas. These plots show that the effect of seasonality in $\beta$ is important. As anticipated, with a temporal window of one year, seasonal vegetation changes are not captured ( $\beta$ estimates remain constant for all initial $t$ ). With a temporal window of six months, slight variations on $\beta$ can be observed. These variations are more pronounced with temporal windows of three and (most variability) one months. Also, note that several nonrobust estimates (depicted as circles and shaded areas) have been obtained predominantly when using one-month temporal windows. The dynamic range of active and passive observations is shown to decrease 

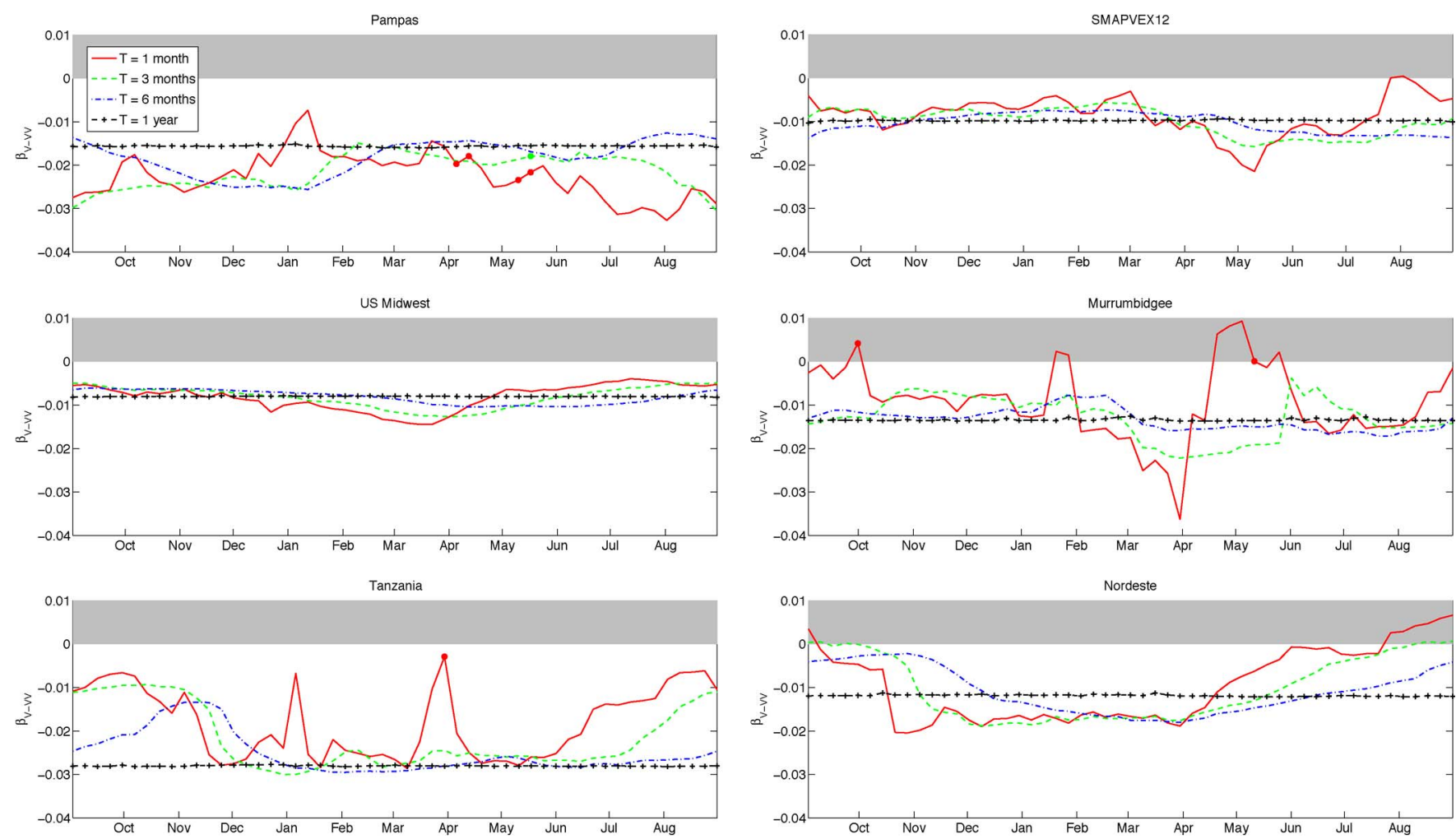

Fig. 10. Slope of Aquarius $e_{V}-\sigma_{V V}$ linear relationship $\left(\beta_{T}(x, t)\right)$ over Pampas, SMAPVEX12, U.S. Midwest, Murrumbidgee, Tanzania, and Nordeste target areas computed using the moving circular window in Fig. 9 with $t=1$ week and $T=1$ month (red solid line), 3 months (green dashed line), 6 months (blue dotdashed line), and 1 year (dotted black line). The shaded area indicates values outside the valid range of $\beta$. Circles indicate statistically insignificant estimates $(p>0.05$ ).

with increasing RVI in Fig. 7 for one year of data. The same behavior is observed when analyzing one, three, and six-month time series of active-passive observations (not shown). Also, the linear dependence of $\beta$ standard error of estimation with land surface heterogeneity shown in Fig. 8 for one-year window length holds when using shorter time series to estimate $\beta$.

To further explore the choice of window length $T, \beta$ is considered to follow vegetation phenology. For a simple annual cycle, most of the spectral density is concentrated in the first and second harmonics. The metric $z$ is introduced as the percent variability contained in the first and second harmonics

$$
z=\frac{c_{1}+c_{2}}{\sum_{i=0}^{\infty} c_{i}},
$$

where $c_{i}$ is the spectral density in the $i$ th harmonic. This metric was applied to the $\beta$ time series calculated using different window lengths $T\left(z_{T}\right)$. By varying the window length, the possible presence of a period in the year which has a distinct and different $\beta$ behavior is examined; the proposed $z$ metric is expected to catch almost any kind of seasonality (smooth sinusoid as well as almost a square or notch). If $T$ is too small, high-frequency noise will remain in the time series, flattening the spectrum and decreasing $z$. If $T$ is too large, a genuine variability in $\beta$ will be averaged out, also decreasing $z$. For instance, in the limit of $T \rightarrow \infty$, all variability will be averaged out, the time series will be a constant, and all of the spectral density will be concentrated in the zeroth harmonic. The maximum $z$ occurs when the chosen window size results in a time series closest to an annual cycle.

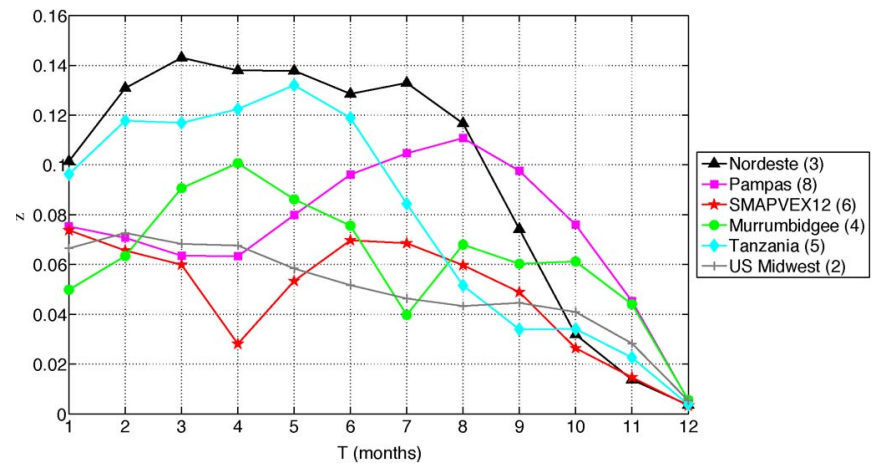

Fig. 11. Plot of percent variability contained in two harmonics (annual and semiannual) for the time series of $\beta$ calculated using the moving circular window in Fig. 9 and $t=1$ week for different window lengths $T\left(z_{T}\right)$ over Pampas, SMAPVEX12, U.S. Midwest, Murrumbidgee, Tanzania, and Nordeste target areas. The maximum $z_{T}$ occurs when the chosen window length results in the $\beta$ time series closest to an annual cycle. The $T$ for the maximum $z_{T}$ is the optimal window size (added in brackets to the legend, in month units).

A plot of $z_{T}$ for different $\beta$ time series—calculated using a moving circular window with $t=1$ week and window sizes $T$-over Nordeste, Pampas, SMAPVEX12, Murrumbidgee, Tanzania, and U.S. Midwest target areas, is shown in Fig. 11. It illustrates that, for every target area, there is an interval of $T$ values that provide relative $z$ maximums and could therefore be appropriate window lengths. Also, the window length $T$ at which the maximum $z$ is reached is geographically dependent. A temporal window of three to six months serves the areas with a highest percentage of canopy cover (Nordeste, Tanzania, and Murrumbidgee), while for croplands (SMAPVEX12 and 
Pampas), a longer window of six to eight months would be necessary. Note that cereals, canola, and corn are the dominant crops in SMAPVEX12, with a seed-to-harvest time of about six months, whereas soybean is the dominant crop in Pampas, having a seed-to-harvest time of around eight months. A shorter window of two months results to be more adequate for U.S. Midwest, which could be due to the high intra-annual variability of grasslands.

The results in Figs. 10 and 11 indicate that the appropriate window length to calculate $\beta$ depends on local effects such as the degree of seasonality in vegetation cover and dynamic variability of soil moisture. Three regimes on the $\beta$ window length have be identified: 1 ) when the window is too small, there are few points in the $e_{V}-\sigma_{V V}$ regression, and there is statistical estimation noise; 2 ) when the window is too large ( $T>9$ months), the $\beta$ estimated does not track the seasonal cycle; and 3) for intermediate window sizes, the window reaches into different parts of the seasonal cycle and causes a phase shift induced by and a function of window size.

It is important to remark that the experimental setup used in this paper does not match the orbit conditions of SMAP (two- to three-day revisit and eight-day exact repeat), with the focus regions having multiple pixels within them and several $e_{V}-\sigma_{V V}$ pairs observed daily. This implies important differences in issue 1), so the $\beta$ estimates obtained for Aquarius cannot be directly applied as the initial values for SMAP. However, results suggest that the SMAP $\beta$ window length could be adapted to dominant land cover, with a maximum of $\sim 2$ months for grasslands and of 6-8 months for croplands.

\section{CONCLUSION}

A sensitivity study of NASA's Aquarius active-passive measurement temporal covariability to land surface characteristics has been performed. The study is relevant to the design of multiresolution active-passive surface soil moisture retrievals in general. Active-passive covariations over short time periods are expected to be mainly due to soil moisture changes; over longer periods (from two to six months), the slope of their temporal linear relationship $(\beta)$ is demonstrated to be a robust parameter depending on local vegetation conditions with seasonally varying phenology. Results show the strength of the temporal linear relationship between spaceborne scatterometer and radiometer observations across major global biomes ( $R^{2}>0.7$ for three months).

Aquarius active and passive observations have a similar spatial resolution. However, radars can make measurements at relatively high spatial resolutions through synthetic aperture processing ( $\sim 3 \mathrm{~km}$ in SMAP). Temporally consistent covariations of active and passive observations as captured by the $\beta$ parameter are key in the baseline SMAP active-passive algorithm that combines radar high spatial resolution and radiometer high sensitivity to soil water content into high-resolution soil moisture fields. While the study here does not provide maps of the parameter that can be used in the SMAP multiresolution retrieval process, the study does provide insights into what landscape and seasonality factors affect the change in the key parameter. An index of landscape heterogeneity (GSI) is intro- duced to quantify and account for land surface heterogeneity within a satellite footprint. Using this metric, the $\beta$ error of estimation is demonstrated to increase with land surface heterogeneity. The RVI is shown to be a good indicator of vegetation scattering, consistent with land cover percent canopy cover. The dynamic range and spread of active-passive observations are shown to decrease with increasing RVI. However, vegetation density (up to moderate densities) does not seem to have an impact on $\beta$ estimation error. The study also addresses the temporal window to estimate $\beta$, which has a minimum given by statistical estimation noise and a maximum depending on the seasonality of the dominant land cover (e.g., $\sim 2$ months for grasslands and 6-8 months for croplands).

\section{REFERENCES}

[1] P. Dubois, J. van Zyl, and E. Engman, "Measuring soil moisture with imaging radars," IEEE Trans. Geosci. Remote Sens., vol. 33, no. 4, pp. 915-926, Jul. 1995.

[2] J. Shi, J. Wang, A. Hsu, P. ONeill, and E. T. Engman, "Estimation of bare surface soil moisture and surface roughness parameter using L-band SAR image data," IEEE Trans. Geosci. Remote Sens., vol. 35, no. 5, pp. 1254-1266, Sep. 1997.

[3] F. Ulaby, R. Moore, and A. Fung, Microwave Remote Sensing Active and Passive. vol. 1 and 2. Norwood, MA, USA: Artech House, 1981.

[4] J. Famiglietti, D. Ryu, A. Berg, M. Rodell, and T. Jackson, "Field observations of soil moisture variability across scales," Water Resources Res., vol. 44, no. 1, pp. 1-16, Jan. 2008.

[5] S. Seneviratne et al., "Investigating soil moisture-climate interactions in changing climate: A review," Earth Sci. Rev., vol. 99, no. 3/4, pp. 125161, May 2010.

[6] D. Entekhabi et al., "An agenda for land surface hydrology research and a call for the second international hydrological decade," Bull. Amer. Meteorol. Soc., vol. 80, no. 10, pp. 2043-2058, Oct. 1999.

[7] W. Krajewski et al., "A remote sensing observatory for hydrologic sciences: A genesis for scaling to continental hydrology," Water Resources Res., vol. 42, no. 7, Jul. 2006, Art. ID. W07301.

[8] Y. Kerr et al., "The SMOS mission: New tool for monitoring key elements of the global water cycle," Proc. IEEE, vol. 98, no. 5, pp. 666-687, May 2010.

[9] D. Entekhabi et al., "The Soil Moisture Active Passive (SMAP) mission," Proc. IEEE, vol. 98, no. 5, pp. 704-716, May 2010.

[10] N. Das, D. Entekhabi, and E. Njoku, "Algorithm for merging SMAP radiometer and radar data for high resolution soil moisture retrieval," IEEE Trans. Geosci. Remote Sens., vol. 49, no. 5, pp. 1504-1512, May 2011.

[11] N. Das et al., "Tests of the SMAP combined radar and radiometer algorithm using airborne field campaign observations and simulated data," IEEE Trans. Geosci. Remote Sens., vol. 52, no. 4, pp. 2018-2028, Apr. 2014.

[12] Y.-Q. Jin and X.-Z. Huang, "Correlation of temporal variations of active and passive microwave signatures from vegetation canopy," IEEE Trans. Geosci. Remote Sens., vol. 34, no. 1, pp. 257-263, Jan. 1996.

[13] A. Colliander, "Analysis of coincident L-band radiometer and radar measurements with respect to soil moisture and vegetation conditions," Eur. J. Remote Sens., vol. 45, no. 1, pp. 111-120, Mar. 2012.

[14] P. Ferrazzoli and L. Guerriero, "Synergy of active and passive signatures to decouple soil and vegetation effects," in Proc. 11th Spec. Meet. MicroRad, 2010, pp. 86-89.

[15] M. Piles, D. Entekhabi, and A. Camps, "A change detection algorithm for retrieving high-resolution soil moisture from SMAP radar and radiometer observations," IEEE Trans. Geosci. Remote Sens., vol. 47, no. 12, pp. 4125-4131, Dec. 2009.

[16] M. Arii, J. van Zyl, and Y. Kim, "A general characterization for polarimetric scattering from vegetation canopies," IEEE Trans. Geosci. Remote Sens., vol. 48, no. 9, pp. 3349-3357, Sep. 2010.

[17] E. H. Simpson, "The measurement of diversity," Nature,vol. 163, no. 688 , pp. 163-168, Apr. 1949.

[18] W. Peake, "Interaction of electromagnetic waves with some natural surfaces," IEEE Trans. Antennas Propag., vol. AP-7, no. 1, pp. 324-329, Dec. 1959.

[19] L. Tsang, J. Kong, and R. Shin, Theory of Microwave Remote Sensing. New York, NY, USA: Wiley, 1985. 
[20] G. Macelloni et al., "Experimental validation of surface scattering and emission models," IEEE Trans. Geosci. Remote Sens., vol. 38, no. 1, pp. 459-469, Jan. 2000.

[21] K. Chen et al., "Emission of rough surfaces calculated by the integral equation method with comparison to three-dimensional moment method simulations," IEEE Trans. Geosci. Remote Sens., vol. 41, no. 1, pp. 90-101, Jan. 2003.

[22] T. Schmugge, P. ONeill, and J. Wang, "Passive microwave soil moisture research," IEEE Trans. Geosci. Remote Sens., vol. GE-24, no. 1, pp. 12-22, Jan. 1986.

[23] T. Mo, B. Choudhury, T. Schmugge, and T. Jackson, "A model for microwave emission from vegetation-covered fields," J. Hydrol., vol. 184, no. C13, pp. 101-129, Dec. 1982.

[24] B. Hornbuckle and A. England, "Diurnal variation of vertical temperature gradients within a field of maize: Implications for satellite microwave radiometry," IEEE Geosci. Remote Sens. Lett., vol. 2, no. 1, pp. 74-77, Jan. 2005.

[25] F. T. Ulaby and M. C. Dobson, Handbook of Radar Scattering Statistics for Terrain. Norwood, MA, USA: Artech House, 1989.

[26] D. M. LeVine, G. S. E. Lagerloef, F. R. Colomb, S. H. Yueh, and F. A. Pellerano, "Aquarius: An instrument to monitor sea surface salinity from space," IEEE Trans. Geosci. Remote Sens., vol. 45, no. 7, pp. 2040-2050, Jul. 2007.

[27] A. B. Smith et al., "The Murrumbidgee soil moisture monitoring network data set," Water Resources Res., vol. 48, no. 7, Jul. 2012, Art. ID. W07701.

[28] H. McNairn et al., "The Soil Moisture Active Passive Validation Experiment 2012 (SMAPVEX12): Prelaunch calibration and validation of the SMAP soil moisture algorithms," IEEE Trans. Geosci. Remote Sens., vol. 53, no. 5, pp. 2784-2801, May 2015.

[29] T. Jackson et al., "Validation of Soil Moisture and Ocean Salinity (SMOS) soil moisture over watershed networks in the U.S." IEEE Trans. Geosci. Remote Sens., vol. 50, no. 5, pp. 1530-1543, May 2012.

[30] D. Entekhabi, N. Das, E. Njoku, J. Johnson, and J. Shi, "Algorithm theoretical basis document L2 \& L3 radar/radiometer soil moisture (active/passive) data products," Jet Propulsion Lab. , California Inst. Technol., Pasadena, CA, USA, Initial Release, v.1, Tech. Rep., 2012.

[31] R. Panciera et al., "The Soil Moisture Active Passive Experiments (SMAPEX): Toward soil moisture retrieval from the SMAP mission," IEEETrans. Geosci. Remote Sens., vol. 52, no. 1, pp. 490-507, Jan. 2014.

[32] K. McColl, D. Entekhabi, and M. Piles, "Uncertainty analysis of soil moisture and vegetation indices using Aquarius scatterometer observations," IEEE Trans. Geosci. Remote Sens., vol. 52, no. 7, pp. 4259-4272, Jul. 2014.

[33] C. W. Gini, "Variability and mutability, contribution to the study of statistical distributions and relations," Studi Economico-Giuridici della Studi Economico-Giuricici della R. Universit'de Cagliari, vol. 3, no. 2, pp. 3-159, 1912.

[34] L. Jost, "Entropy and diversity," Oikos, vol. 113, no. 2, pp. 363-375, May 2006

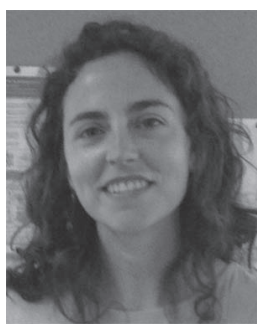

María Piles (S'05-M'11) received the B.S. and M.S degrees in telecommunication engineering from the Universitat Politècnica de València (UPV), València, Spain, in 2005 and the Doctoral degree from the Universitat Politècnica de Catalunya (UPC), Barcelona, Spain, in 2010

She joined the University of Melbourne, Parkville, Vic., Australia, as a Research Fellow in 2010. She is currently a Research Scientist with UPC, based at the SMOS Barcelona Expert Center. She is also a Research Affiliate with the Department of Civil and Environmental Engineering, Massachusetts Institute of Technology (MIT), Cambridge, MA, USA, through the MIT MISTI Global Seed Fund program. Her research activity is centered in remote sensing for Earth observation, with special emphasis on radiometers, radars and visible/infrared sensors, retrieval of soil moisture and microwave vegetation parameters, and development of data fusion techniques.

Dr. Piles received the Med-Storm Prize for Young Researchers in the Plinius Conference (2011) and the UPC Special Doctoral Award in Information Technology and Communication (2012). Since 2012, she is a board member of the IEEE Geoscience and Remote Sensing Society, Spanish section.

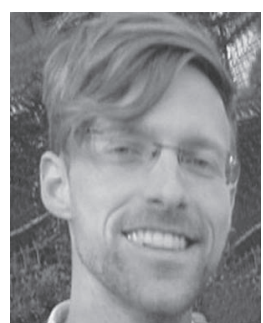

Kaighin A. McColl (S'09) received the Bachelor of Environmental Engineering (Hons) and Bachelor of Science (applied mathematics) degrees from the University of Melbourne, Parkville, Vic., Australia, in 2009. He is currently working toward the Ph.D. degree in the Department of Civil and Environmental Engineering, Massachusetts Institute of Technology, Cambridge, MA, USA.

His research interests lie in boundary-layer meteorology, land-atmosphere interactions, and remote sensing of soil moisture and vegetation. He is funded by the National Science Foundation's Graduate Research Fellowship Program.

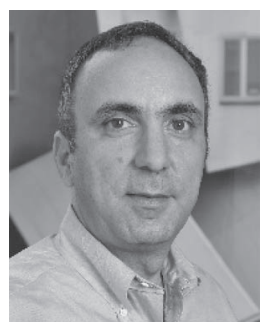

Dara Entekhabi (M'04-SM'09-F'15) received the B.S. and M.S. degrees from Clark University, Worcester, MA, USA, and the Ph.D. degree from the Massachusetts Institute of Technology (MIT), Cambridge, MA, USA, in 1990

$\mathrm{He}$ is currently a Professor with the Department of Civil and Environmental Engineering and the Department of Earth, Atmospheric and Planetary Sciences, MIT. He is the Science Team Lead of the National Aeronautics and Space Administration's Soil Moisture Active and Passive mission that was launched on January 31,2015 . His research includes terrestrial remote sensing, data assimilation, and coupled land-atmosphere system modeling.

Prof. Entekhabi is a Fellow of the American Meteorological Society and the American Geophysical Union.

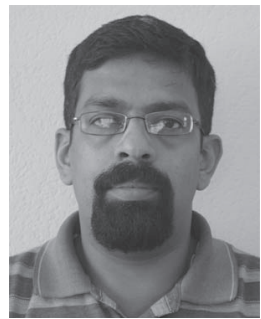

Narendra Das received the B.Eng. degree from the National Institute of Technology (formerly Government Engineering College), Raipur, India, and the Master's and Ph.D. degrees from Texas A\&M University, College Station, TX, USA, in 2005 and 2008, respectively.

He joined the Jet Propulsion Laboratory (JPL), Pasadena, CA, USA, in 2008, and he has been conducting research in hydrology and microwave remote sensing research on land. He is currently a member of the Water and Carbon Cycles Group, Climate, Oceans, and Solid Earth Science Section, JPL. He is also a member of the Science Algorithm Development Team for the Soil Moisture Active and Passive mission.

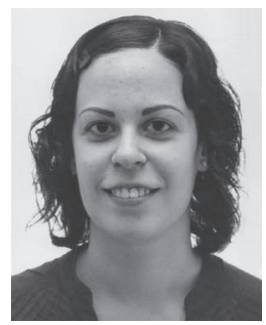

Miriam Pablos (S'14) received the Telecommunication Engineering degree and the M.S. degree from the Universitat Politècnica de Catalunya (UPC), Barcelona, in 2010 and 2012, respectively, where she is currently working toward the Ph.D. degree, with an FPI Grant from the Spanish Ministry of Economy and Competitiveness.

In 2009, she joined the Remote Sensing Laboratory Group of the Department of Signal Theory and Communications, UPC, involved in the ESA's Soil Moisture and Ocean Salinity (SMOS) mission. Since 2012, she is a member of the SMOS Barcelona Expert Center (SMOS-BEC), where she is working on remote sensing devoted to Earth observation, using several L-band sensors such as SMOS and Aquarius. Her current research interest areas include the assessment of the SMOS and Aquarius brightness temperature products, the investigation of the Antartica's emissivity through the analysis of SMOS and Aquarius observations, and the study of soil moisture and vegetation. 